\title{
Computationally Implemented Modeling of Creep of Compo- site Materials Caused by Phase Dissolution
}

\author{
Xiaodan $\mathrm{Li}^{\mathrm{a}}$, Syeda Rahman ${ }^{\mathrm{a}}$ and Zachary C. Grasley ${ }^{\mathrm{a} *}$ \\ a. Texas A\&M University, College Station, TX USA \\ *Corresponding author.Email: zgrasley@tamu.edu
}

\begin{abstract}
Multiphasic composites with time-evolving, transient microstructures exhibit time-dependent behavior under load. One mechanism leading to this behavior is the dissolution of loadbearing phases within such materials. When the dissolution process occurs inside one phase of the composite material, the stress transmitted by the dissolving phase transfers into the neighboring phases, resulting in additional deformation of the whole composite. This additional deformation of the macroscopic material manifests as time-dependent creep of the composite. The purpose of this paper is to provide a general kinematic framework for such dissolution induced creep for virtually any material class, and to mechanistically model the same utilizing a computationally-implemented approach that is consistent with the kinematic framework. Utilizing this modeling approach, two distinct processes are computationally simulated, namely 1) creep of a water saturated, porous, frozen body upon warming and 2) creep of cement paste due to hydration-induced dissolution of cement grains. The simulation results show that microstructure evolution induces significant creep behavior of the whole composite in these two simulated cases.
\end{abstract}

Keywords: Microstructure; Dissolution; Computational; Finite Element Method; Viscoelastic; Creep

\section{Introduction}

Many natural and man-made materials are multiphasic composite materials, such as polymers, mortar and concrete, alloys, porous media, etc. Most of these materials exhibit notable time-dependent viscoelastic/viscoplastic (VE/VP) behavior under long-term external mechanical loading, and many computational algorithms and numerical simulation techniques have been developed to study the creep/relaxation behavior of composite materials, such as mesoscale finite element simulations of concrete [1], micromechanical modeling approaches of polymers [2], constitutive derivations of nanocomposite melts [3], etc. Research has also been conducted to study the mechanisms behind the VE/VP behavior of each material, and it has been proposed that dissolution of load-bearing phases occurring during the microstructure evolution plays an important role in inducing time-dependent deformation behavior of the macroscopic materials. This dissolution can be caused by 
chemical reaction inside the material, e.g., cementitious materials [4], phase transformations, or 'pressure solution' effects [5-8], e.g., quartz [9, 10] and olivine [11]. Despite the importance of the phase dissolution mechanism with respect to creep and relaxation, there currently is no holistic kinematical framework and corresponding computational scheme to support modeling transient deformation associated with the dissolution-formation process. Recently, a computational scheme has been developed to give predictions of the overall VE/VP behavior of the multiphasic composites. This computationally-implemented model couples a microstructure model, which give time-evolving microstructures of the composites, and a time-stepping finite element model, which calculates the stress/strain fields inside a material as a function of evolving microstructures [12-14]. The dissolution process of load-bearing phases is simulated in the computational scheme, and one can investigate the VE/VP responses of composites directly from the simulation results. Through inputting different time-evolving microstructures of a material into the model within this computational scheme, the redistribution of stress and strain fields occurring inside the composite as a result of microstructure evolution can be simulated, and thus the creep/relaxation process modeled. The kinematical framework and modeling approach are general enough to even model the evolution of stress fields (and resulting creep) in diffusive creep problems (e.g., [15]) where the diffusing species could be treated as a dissolving/re-precipitating phase.

The objective of this paper is first to establish a holistic kinematic framework linking the microstructure evolution to the evolution of stress and strain fields to better understand the evolution of composite constitutive properties and deformation mechanisms. The second objective is to demonstrate the utility and robustness of the framework and affiliated modeling approach via prediction of the dissolution induced creep of two disparate classes of materials. In particular, the computational scheme is applied to both a porous, water-saturated, frozen body when subjected to warming and hydrating cement paste, such that the effects of phase dissolution on creep can be demonstrated according to the simulation results.

\section{Computationally implemented model and framework}

\subsection{Conceptualization and kinematic framework}

The computational scheme integrates two different models: a time-evolving microstructure model and a finite element based mechanical model. The microstructure models generate time-evolving microstructures for different composite materials, which are then utilized in the finite element calculation model to predict the time-evolving creep and elastic responses of these composites.

To account for the time dependence (as well as the strain and stress history dependence) of the elastic and viscoelastic properties, the computational scheme is discretized in time. At each time step in the computational scheme, the microstructure models provide a 3D snapshot of the composite materials for the corresponding age of the time step. All the 3D snapshots of the materials are meshed into voxels utilizing a spatially aligned numerical discretization [16]. Thus, each voxel is an eight node cubic finite element comprised of a single homogenous material. Therefore, in the computational-implemented model, the evolution of the microstructure with time occurs at the scale of the voxels. Then, finite element 
calculations are carried out on these meshed 3D time-evolving microstructures to predict the time-evolving mechanical responses of these composites under periodic strain-controlled boundary conditions. The evolving mechanical properties are subsequently calculated via the spatially averaged stress and strain fields. At each time step, phase change may occur in any of the voxels as prescribed by the evolution of the microstructure from one time step to the next- including both solid, load-bearing phases and phases that may not transmit stress (e.g., gases). Regarding conservation of linear momentum, it is assumed that once phase change occurs in one voxel, the stress being transmitted by this voxel would be transferred into surrounding voxels. Meanwhile, the newly formed phase in this voxel is assumed to be infinitely compliant such that it would not carry any stress at the time of formation, but meanwhile its configuration conforms to the preexisting deformed voxel, neglecting all the memories of historical responses. This procedure supposes that the process of stress redistribution takes much less time than microstructure evolution itself. This time-dependent stress redistribution is elaborated conceptually in Fig. 1 under a stresscontrolled boundary condition.

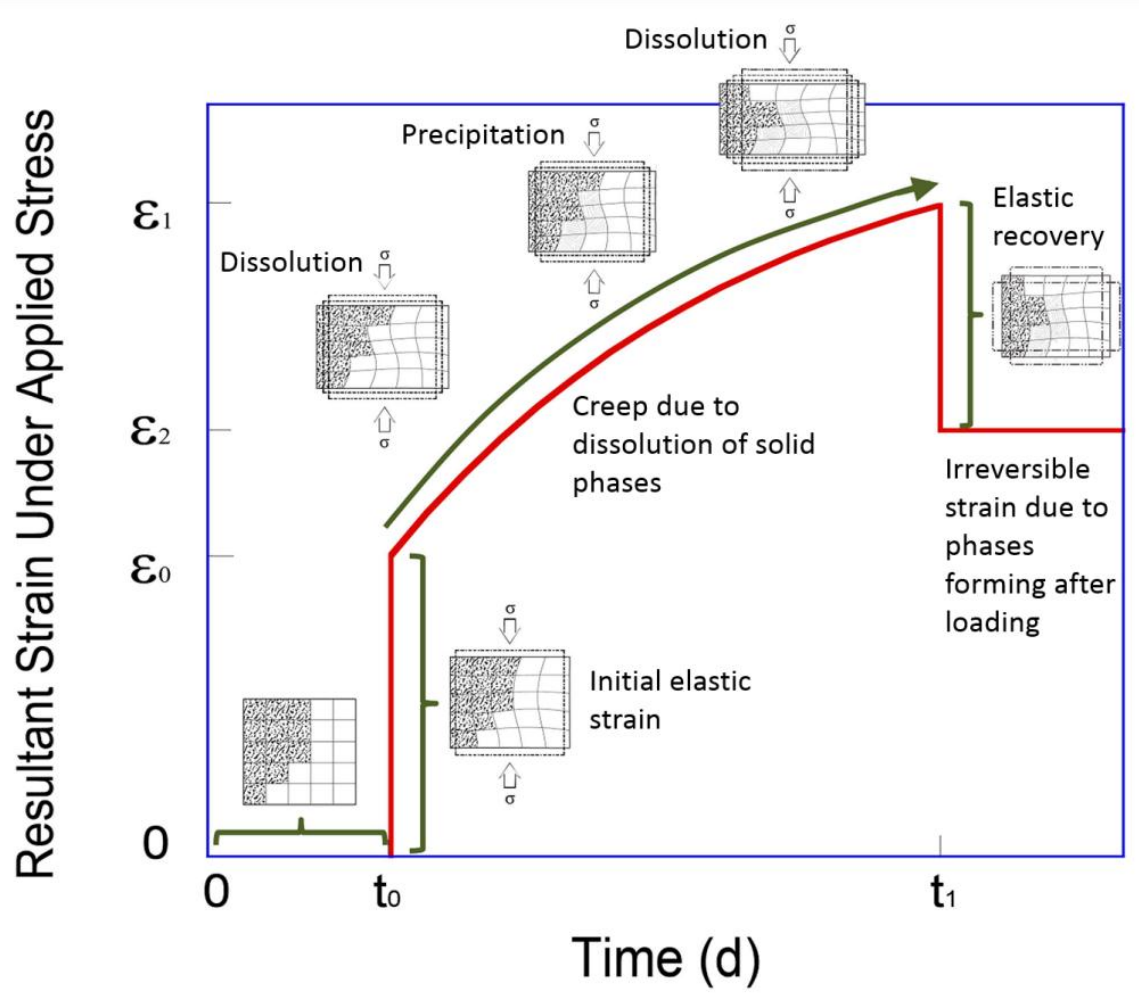

Fig. 1. Conceptual diagram of the computational scheme. The creep behavior of an evolving microstructure is shown in the figure, with $\sigma$ denoting a controlled external macroscale load. (a) Initially, load-bearing phases exist inside the composite before load is applied. (b) When an external load is applied at time $t_{0}$, the body deforms elastically immediately. (c) As load-bearing phases dissolve in certain voxels, the stress transmitted by the surrounding solid voxels increases, increasing the elastic deformation in these surrounding voxels, leading to an increase in the macroscopic deformation of the whole composite. Since the rate of stress transfer depends on dissolution rate, this results in creep. (d) New phases form with their configurations conforming to the deformed voxels while carrying no stress. (e)This process repeats as more material dissolves and 
the whole composite deforms again. (f) When the external load is removed at time $t_{1}$, the body deforms elastically again, but it will not recover back to its initial configuration, leaving a permanent irreversible strain.

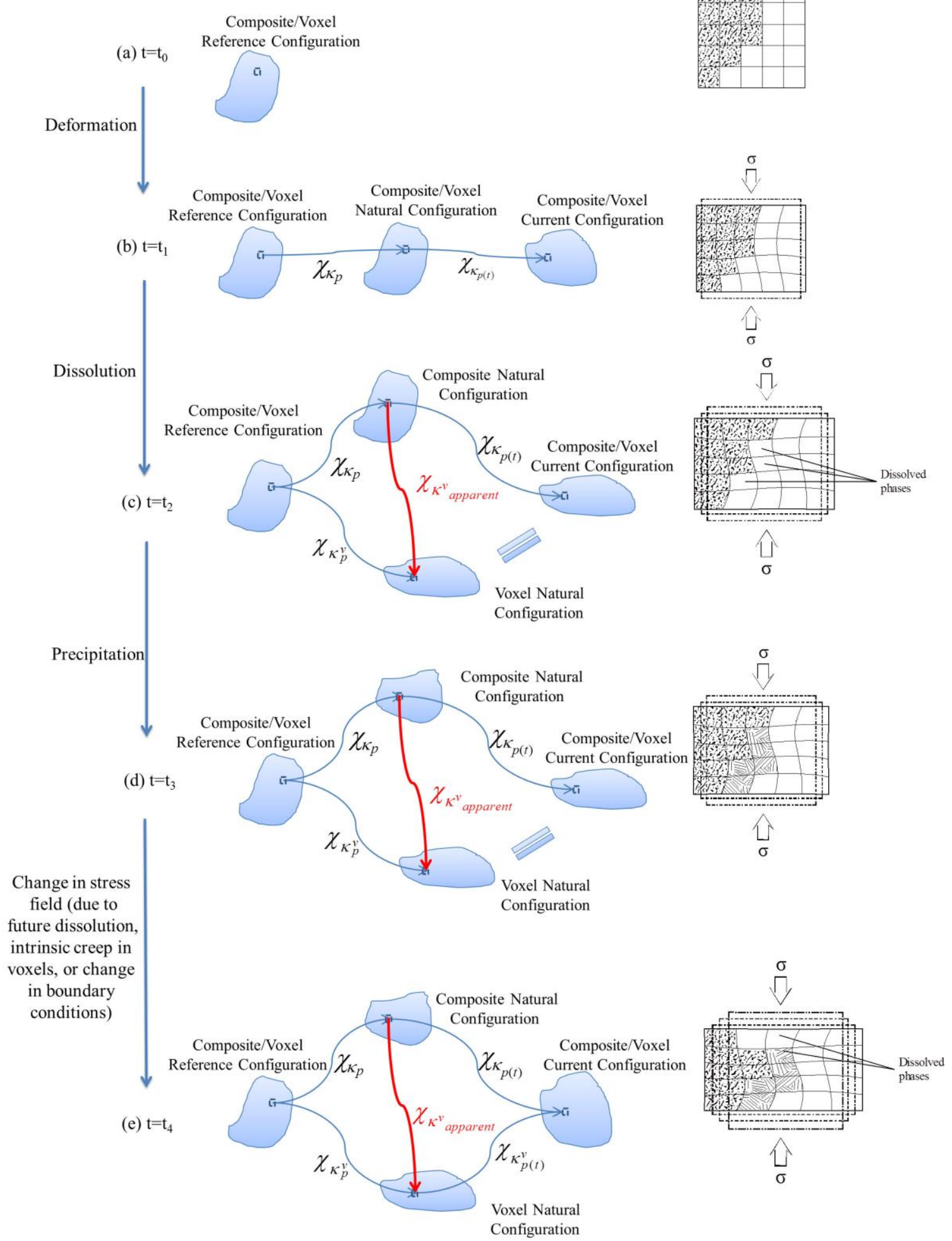


Fig. 2 Overview of the kinematical framework of the dissolution-formation process.

The concept of evolving natural configurations [17-19] can be utilized to develop a consistent kinematical framework to quantify the effect of dissolution of load bearing phases on the equilibrium state of composite materials. When a material is subjected to an external stimuli (or multiple stimuli) such as an external traction, temperature change, or moisture change, the material body will deform from the reference configuration to the current configuration. If the external stimuli are removed from the material body, it will deform back to its natural configuration; for the simplest, purely elastic bodies, the natural configuration may coincide with the reference configuration. In the case of the material with dissolving solids, as dissolution processes progress, under any external or internal stimuli (e.g. applied tractions/displacements), the composite body - as well as every voxel comprising the composite - deform from the initial, reference configuration to the current configuration. As the dissolution-formation process occurs over the same time scale as the motion, with the removal of external stimuli the composite will not return back to the reference configuration, but rather to the composite natural configuration, which may evolve with time. It has been demonstrated that the evolution of natural configurations could lead to creep in purely elastic bodies [20], and this is the basis of the hypothesized expectation that dissolution effects would lead to creep of composites. The same evolution of configurations that happens with the composite as a whole can be presumed to likewise occur with any voxel. Since the natural configuration for the composite can be different from the natural configuration for any given voxel at a given time ${ }^{1}$, this difference in motion yields "apparent strain" in the composite natural configuration, as shown in Fig. 2.

Fig. 2 shows the overview of the kinematical framework of the dissolution-formation process utilizing the concept of time-evolving natural configurations. Note that conceptually, this framework is analogous to that utilized in the analysis of mixtures or other materials with multiple natural configurations [21-27]. In Fig. 2, the framework at each step corresponds to one time step shown in Fig. 1. The motions $\chi_{\kappa}$ show the paths of a material point between different configurations. The motions $\chi_{\kappa_{p}}$ and $\chi_{\kappa_{p(t)}}$ denote the path of a point in the composite body from the initial, reference configuration to the natural configuration of the composite, and then to the current configuration. The motions $\chi_{\kappa_{p}^{v}}$ and $\chi_{\kappa_{p(t)}^{v}}$ denote the motion of a given voxel (occupying the same location in the composite as the point referred to above) from the initial reference configuration to the natural configuration, and then to the current configuration. In Fig. 2(a), all the motions (both the composite and voxel) start from the initial reference configuration. At $t=t_{1}$, as shown in Fig. 2(b), external or internal stimuli are applied on the material, deforming the composite body from the reference configuration to the current configuration, and removal of the stimuli will not necessarily make the composite return back to the initial reference configuration. If the inherent phases that comprise the composite are VE/VP themselves, the natural configuration of the whole composite may evolve with time, and with the removal of the stimuli, the body will return to the evolved natural configuration instead of the reference configuration;

\footnotetext{
${ }^{1}$ The natural configuration of an individual voxel should be interpreted as the configuration that voxel would return to if any stimuli applied to that individual voxel were removed (e.g., if applied stress were removed from the voxel).
} 
likewise for the individual voxels inside the microstructure. Once phase dissolution occurs in a voxel at $t=t_{2}$, as shown in Fig. 2(c), any stress carried by this voxel is forced to be zero and the current configuration becomes the voxel's new natural configuration. At this time, before the formation of new phases, the natural configuration of the composite stays the same as the configuration shown in Fig. 2(b), leading to a difference between the composite natural configuration and the voxel natural configuration. This difference in deformation is a mapping $\chi_{\kappa_{\text {apparent }}^{v}}$ that leads to "apparent strain" in the composite body. From the perspective of continuum mechanics, apparent strain is defined here as the strain that exists independent of any applied stimuli (the state of stress specifically in this paper). At $t=t_{3}$ in Fig. 2(d), the natural configuration of the composite changes again with the formation or precipitation of new solid phases. At the last time step in Fig. 2(e), with any future change in the stress field within the composite (which may occur due to further dissolution, intrinsic creep in the voxels, and/or change in boundary conditions), voxels filled with newly formed phases deform from the individual voxel natural configuration to the new current configuration; likewise, the whole composite body deforms to the new current configuration.

From Fig. 2, it can be seen that the existence of apparent strain is a key reason behind the dissolution-induced VE/VP behavior and the irreversible nature of much of the dissolution induced creep. To computationally predict the overall deformation behavior of the composite material, the effect of apparent strain on the inherent stress and strain fields inside the composite must be quantified. Fig. 3 shows a more complete version of the framework demonstrated in Fig. 2(e), and in this figure, the motion of a material point inside continuous body is defined as

$$
\mathbf{x}=\chi_{\kappa}(\mathbf{X}, t)
$$

where $\mathbf{x}$ is the position vector in the current configuration of the motion, $\mathbf{X}$ is the position vector in the reference configuration of the motion, and the boldface indicates a vector (or, in general, a higher order tensor). The deformation gradient of the body is defined according to

$$
\mathbf{F}=\frac{\partial \mathbf{x}}{\partial \mathbf{X}}
$$




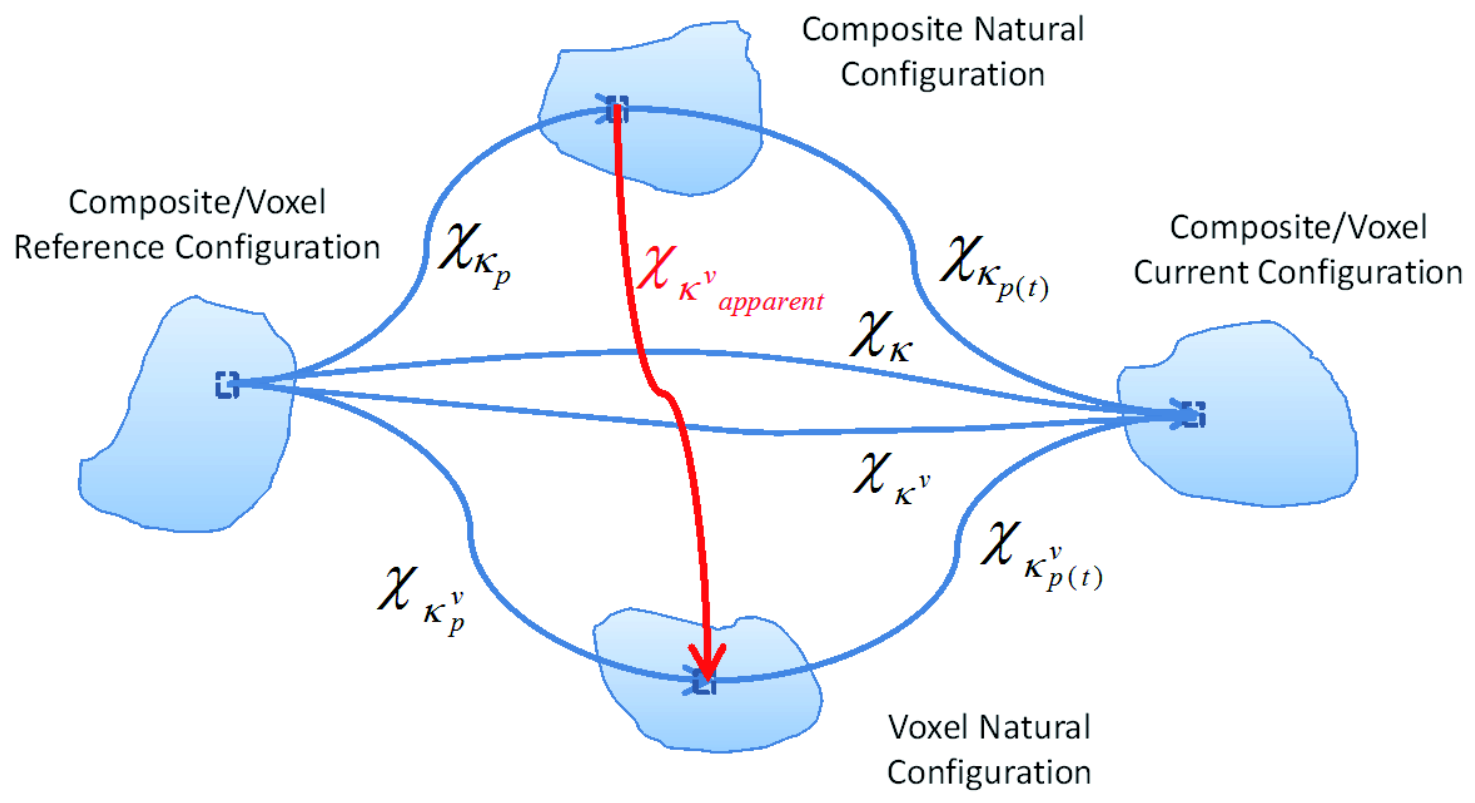

Fig. 3 A complete view of the kinematic framework shown in Fig. 2(e).

As stated previously, a primary goal here is to quantify the stress relaxation or creep that occurs due to phase dissolution in a composite microstructure. Such stress relaxation or creep is determined by quantifying the time evolution of the spatially averaged strain field and the spatially averaged stress field under a particular boundary condition. For a composite material body input into the computational scheme, spatially averaged strain is calculated according to the deformation from the reference configuration to the current configuration (i.e., motion $\chi_{\kappa}$ ). The spatially averaged stress field is calculated according to the difference between the composite natural configuration and the composite current configuration, which is mapped through the motion $\chi_{\kappa_{p(t)}}$. Unfortunately, when stress relaxation or creep is induced by dissolution of load-bearing phases, one cannot directly determine the deformation gradient (and thus the strain and stress) associated with the motion $\chi_{\kappa_{p(t)}}$. However, the motion $\chi_{\kappa_{p(t)}}$ can be decomposed into two different computable motions: one from the composite natural configuration to the voxel natural configuration $\chi_{\kappa_{\text {apparent }}^{v}}$, and one from the voxel natural configuration to the current configuration $\chi_{\kappa_{p(t)}^{v}}$. For these three motions, according to eq. (2), the deformation gradients are 


$$
\begin{aligned}
& \text { motion } \chi_{\kappa_{p(t)}}: \mathbf{F}_{\kappa_{p(t)}}=\frac{\partial \mathbf{x}_{\kappa}}{\partial \mathbf{x}_{\kappa_{p}}} \\
& \text { motion } \chi_{\kappa_{a p p a r e n t}^{v}}: \mathbf{F}_{\kappa_{a p p a r e n t}^{v}}=\frac{\partial \mathbf{x}_{\kappa_{p}^{v}}}{\partial \mathbf{x}_{\kappa_{p}}}, \\
& \text { motion } \chi_{\kappa_{p(t)}^{v}}: \mathbf{F}_{\kappa_{p(t)}^{v}}=\frac{\partial \mathbf{x}_{\kappa^{v}}}{\partial \mathbf{x}_{\kappa_{p}^{v}}}=\frac{\partial \mathbf{x}_{\kappa}}{\partial \mathbf{x}_{\kappa_{p}^{v}}}
\end{aligned}
$$

where $\mathbf{x}_{\kappa}$ and $\mathbf{x}_{\kappa^{v}}$ are the position vectors of a point in the composite body and the corresponding voxel in the current configuration, $\mathbf{x}_{\kappa_{p}^{v}}$ is the position vector of the voxel in the voxel natural configuration, and $\mathbf{x}_{\kappa_{p}}$ is the position vector of the point corresponding to the location of the voxel in the composite natural configuration. From eq. (3), one can derive that

$$
\mathbf{F}_{\kappa_{p(t)}}=\mathbf{F}_{\kappa_{\text {apparent }}^{v}} \mathbf{F}_{\kappa_{p(t)}^{v}} .
$$

Combining eq. (4) with the Green-Lagrangian strain expression

$$
\mathbf{E}=\frac{1}{2}\left(\mathbf{F}^{T} \mathbf{F}-\mathbf{I}\right)
$$

where $\mathbf{I}$ is the identity tensor, one finds that for the motion $\chi_{\kappa_{p(t)}}$,

$$
\mathbf{E}_{\kappa_{p(t)}}=\frac{1}{2}\left(\left(\mathbf{F}_{\kappa_{\text {apparent }}^{v}} \mathbf{F}_{\kappa_{p(t)}^{v}}\right)^{T}\left(\mathbf{F}_{\kappa_{\text {apparent }}^{v}} \underset{\mathbf{F}_{p(t)}^{v}}{ }\right)-\mathbf{I}\right)=\frac{1}{2}\left(\mathbf{F}_{\kappa_{p(t)}^{v}}{ }^{T} \mathbf{F}_{\kappa_{\text {apparent }}^{v}}{ }^{T} \mathbf{F}_{\kappa_{\text {apparent }}^{v}} \mathbf{F}_{\kappa_{p(t)}^{v}}-\mathbf{I}\right),
$$

where $\mathbf{E}_{\kappa_{p(t)}}$ is the strain tensor of a particular point in the composite body generated during the motion $\chi_{\kappa_{p(t)}}$ from the composite natural configuration to the current configuration. Similarly, according to eq. (5), for motion $\chi_{\kappa_{a p p a r e n t}^{v}}$ and $\chi_{\kappa_{p(t)}^{v}}$,

$$
\mathbf{E}_{\kappa_{\text {apparent }}^{v}}=\frac{1}{2}\left(\mathbf{F}_{\kappa_{\text {apparent }}^{v}}{ }^{T} \mathbf{F}_{\kappa_{a p p a r e n t}^{v}}-\mathbf{I}\right) \text { and } \mathbf{E}_{\kappa_{p(t)}^{v}}=\frac{1}{2}\left(\mathbf{F}_{\kappa_{p(t)}^{v}{ }^{T} \mathbf{F}_{\kappa_{p(t)}^{v}}}-\mathbf{I}\right)
$$

where $\mathbf{E}_{\kappa_{\text {apparent }}^{v}}$ is the strain tensor for the voxel that exists at the same, particular point in the composite, generated during the motion $\chi_{\kappa_{a p p a r e n t}^{v}}$, and $\mathbf{E}_{\kappa_{p(t)}^{v}}$ is the strain tensor of the particular voxel generated during the motion $\chi_{\kappa_{p(t)}}$.

Combining (6) and (7) results in

$$
\mathbf{E}_{\kappa_{p(t)}}=\mathbf{F}_{\kappa_{p(t)}^{v}}{ }^{T} \mathbf{E}_{\kappa_{a p p a r e n t}^{v}} \mathbf{F}_{\kappa_{p(t)}^{v}}+\mathbf{E}_{\kappa_{p(t)}^{v}}
$$


We now recognize that the displacement

$$
\mathbf{u}_{\kappa_{p}^{v}}=\mathbf{x}_{\kappa}-\mathbf{x}_{\kappa_{p}^{v}}
$$

is the displacement of a given voxel from the natural configuration of the voxel to the current configuration. Since

$$
\mathbf{F}_{\kappa_{p(t)}^{v}}=\frac{\partial \mathbf{x}_{\kappa}}{\partial \mathbf{x}_{\kappa_{p}^{v}}}=\operatorname{Grad}_{\kappa_{p}^{v}} \mathbf{u}_{\kappa_{p}^{v}}+\mathbf{I},
$$

where one should carefully note that $\operatorname{Grad}_{\kappa_{p}^{v}}$ is the gradient operation in reference to the position in the voxel natural configuration, we find that (8) becomes

$$
\mathbf{E}_{\kappa_{p(t)}}=\left(\operatorname{Grad}_{\kappa_{p}^{\nu_{p}}} \mathbf{u}_{\kappa_{p}^{v}}^{T}+\mathbf{I}\right) \mathbf{E}_{\kappa_{\text {apparent }}^{v}}\left(\operatorname{Grad}_{\kappa_{p}^{v}} \mathbf{u}_{\kappa_{p}^{v}}+\mathbf{I}\right)+\mathbf{E}_{\kappa_{p(t)}^{v}}
$$

If the strains are small in magnitude (as in the simulations considered in this work), then (11) becomes

$$
\boldsymbol{\varepsilon}_{\kappa_{p(t)}}=\boldsymbol{\varepsilon}_{\kappa_{a p p a r e n t}^{v}}+\boldsymbol{\varepsilon}_{\kappa_{p(t)}^{v}}
$$

where $\varepsilon$ is the infinitesimal, linearized strain. Eq. (12) may also be written as

$$
\boldsymbol{\varepsilon}=\boldsymbol{\varepsilon}_{\text {apparent }}+\boldsymbol{\varepsilon}_{\text {mechanical }},
$$

where $\boldsymbol{\varepsilon}_{\text {apparent }}$ is the apparent strain of a particular voxel generated during the dissolutionformation process (and associated with the mapping of a point from the composite natural configuration to the corresponding voxel natural configuration), and $\boldsymbol{\varepsilon}_{\text {mechanical }}$ is the mechanical strain of the voxel as a result of the stress state within that voxel. Note that the specific requirement for the use of the linearized strain here is that the individual voxel strain between the natural and current configurations must be small along with the product of that strain and the apparent strain that occurs between the composite body as a whole and the individual voxels. It is important to be aware that one might have a composite exhibiting small strain at the macroscopic length scale while yet exhibiting locally large strains at the voxel level (depending on choice of length scale for the voxel); in such a case, the linearization in (12) is inappropriate.

From eq. (13), it can be seen that, from a book-keeping perspective, apparent strain can be treated in the same fashion as free strains or eigenstrains [28], except that the apparent strain does not involve a change in the atomic or molecular spacing from the reference configuration, but a change from the natural configuration of the composite body to the natural configuration of a specific voxel. Other free strains that are induced by temperature change or internal relative humidity change are produced as a result of the configuration change from the reference configuration to the natural configuration of the composite. In the computational scheme, when calculating the stress in a particular voxel, only the mechanical strain is involved in the calculation while the stress associated with the apparent 
strain is strictly zero. Since all the microscopic phases in the composites simulated later in this paper are strictly linearly elastic, the apparent VE/VP effects of the composites occurs purely due to microstructure evolution, and this effect of load bearing solids dissolution can be substantial.

To implement the above conceptualization and kinematic framework of the dissolution process into the computational scheme, the phases inside each voxel at all time steps should be tracked and all the voxels that participate in the dissolution process should be recorded. In the model, under the applied strain controlled periodic boundary condition over the entire time scale, different microstructures of composites are input into the scheme. Microstructures from two neighboring time steps would be compared first at the beginning of each time step, so that once the phases in one single voxel changes between the two time steps, the location and the configuration of this voxel would be noted for further calculation, while its historical mechanical responses would be expunged. The newly formed phases would be assigned with new constitutive properties for the subsequent time steps. Finite element analysis is carried out on the new microstructure to calculate the new internal stress and strain fields inside the material, and this procedure repeats for all time steps. Apparent VE/VP relaxation moduli are determined by quantifying the volume averaged stress at each time step (recall that the volume averaged strain is fixed as zero to simulate a relaxation experiment). More detailed information regarding the conceptualization of the model can be found in $[12,13]$.

\subsection{Finite element calculation}

A computationally implemented homogenization model utilizing the finite element method was developed previously to predict the elastic moduli of 3D composites [29-31]. The new computational scheme discussed herein was developed based on this existing elastic moduli prediction model while utilizing time-evolving microstructures as input and including the process and stress redistribution effects of dissolution. As mentioned previously, the concept of time steps was used in this computational scheme to account for the stress/strain history dependent mechanical responses of the material. Thus, for every time step in the model, for the composite microstructure to reach a mechanical equilibrium state, the total mechanical energy stored in the whole microstructure should be minimized $[12,13]^{2}$. Once the mechanical equilibrium state is reached, the volume averaged stress may be predicted through solving a series of elastic equations on the finite element mesh [32]. Then the composite moduli at this time step can be calculated with the predicted volume averaged stress and the known applied controlled strain.

At any time step, since all the phases inside the microstructure voxels are purely linearly elastic, when the composite is subject to infinitesimal strains, the total elastic mechanical energy stored in one single voxel can be calculated by

\footnotetext{
${ }^{2}$ We disregard any inertia in the body in this solution, which results in minimal error since the velocity of the deformation is very slow under the prescribed boundary conditions.
} 


$$
E n=\frac{1}{2} \iint_{0}^{1} \int_{0}^{1} \int_{0}^{1} \varepsilon_{p q} C_{p q r s} \varepsilon_{r s} d x d y d z,
$$

where $E n$ is the total stored elastic energy, $\varepsilon_{p q}$ is the infinitesimal strain tensor, $C_{p q r s}$ is the elastic moduli tensor; $p, q, r, s=1,2$, or 3 , and the integral is over the volume of a single unit voxel. By expressing the strain tensor in terms of displacement components, eq. (14) can be rewritten as

$$
E n=\frac{1}{2} u_{r p}^{T} D_{r p s q} u_{s q},
$$

where $D_{r p s q}$ is the stiffness matrix and $u_{r p}$ is the $p$ 'th component of displacement at $r$ 'th node. From eq. (13), the strain tensors for any voxel in the microstructure can be approximated as the sum of the mechanical strain and the apparent strain, and so may the displacement vector of the nodes,

$$
u_{r p}=U_{r p}+\delta_{r p}+\delta_{r p}^{a p p a r e n t}
$$

where $U_{r p}$ is the displacement vector determined by the surrounding voxels, $\delta_{r p}$ is the correction vector determined by periodic boundary conditions, and $\delta_{r p}^{\text {apparent }}$ is the correction vector determined by the apparent strain produced in the microstructure during the dissolution process. Defining $\delta_{r p}^{\text {combined }}=\delta_{r p}+\delta_{r p}^{\text {apparent }}$, eq. (15) becomes

$$
E n=\frac{1}{2}\left[u_{r p}^{T} D_{r p, s q} u_{s q}+2 \delta_{r p}^{\text {combined }} D_{r p, s q} u_{s q}+\delta_{r p}^{\text {combined }} D_{r p, s q} \delta_{s q}^{\text {combined }}\right]
$$

where $\delta_{r p}^{\text {combined }}$ is the total correction vector for the displacement of a node. Eq. (17) can be simplified as

$$
E n=\frac{1}{2} \mathbf{u A u}+\mathbf{b u}+C
$$

where $\mathbf{A}$ is the Hessian matrix comprised of the stiffness matrices, $\mathbf{u}$ is a vector of all the displacements, $\mathbf{b}$ is a global vector, and $C$ is a global constant; $\mathbf{b}$ and $C$ are determined by both the external strain controlled, periodic boundary conditions, and the dissolution and precipitation process of phases that occurs in voxels, which generates apparent strain. Since in eq. (18) there is no term with an order higher than quadratic, to derive the exact displacement solution leading to the minimum elastic energy, Simpson's rule may be used such that

$$
\frac{\partial E n}{\partial \mathbf{u}}=\mathbf{A u}+\mathbf{b}=0
$$

In the computational scheme, the apparent VE/VP bulk modulus and VE/VP shear modulus are calculated directly from the simulation results when the composites are subjected to prescribed, periodic volumetric strain and prescribed, periodic shear strain. 
Due to a constraint in the current version of the computational scheme, microstructures that are input directly into the model can only be subjected to strain-controlled periodic boundary conditions. To predict the time-evolving strain of the macroscopic composite under constant external stress, Boltzmann's superposition principle [33, 34] was used to simulate the constant, stress-controlled periodic boundary conditions. First, the spatially averaged hydrostatic stress of the composite under constant unit periodic volumetric strain may be calculated as a function of time for different loading ages; due to dissolution, this stress relaxes with time. In order to maintain an overall spatially averaged constant stress state, a necessary amount of extra strain must be applied on the composite at each time step. This additional strain may be calculated according to the superposition principle via

$$
\begin{aligned}
& \text { if } i=1, \varepsilon\left(t_{i}\right)=\frac{-\sigma_{0}}{3 K_{t_{i}}\left(t_{i}\right)} ; \\
& \text { if } i \neq 1, \varepsilon\left(t_{i}\right)=\frac{\sigma_{0}-\sum_{k=1}^{i-1}\left[\varepsilon\left(t_{k}\right) K_{t_{k}}\left(t_{i}\right)\right]}{3 K_{t_{i}}\left(t_{i}\right)},
\end{aligned}
$$

where $\sigma_{0}$ is the maintained constant boundary stress, $\varepsilon\left(t_{i}\right)$ is the additional linear strain to be applied on the composite at time $t_{i}$ to achieve the desired boundary stress history, and $K_{t_{k}}\left(t_{i}\right)$ is the apparent VE/VP bulk modulus of the composite at time $t_{i}$ when loaded at age $t_{k}$. The resultant strain history of the composite (e.g., the sum of the creep and elastic strains) is calculated through

$$
\varepsilon_{\text {total }}\left(t_{i}\right)=\sum_{k=1}^{i} \varepsilon\left(t_{k}\right)
$$

where $\varepsilon_{\text {total }}\left(t_{i}\right)$ is the strain of the composite at time $t_{i}$. The preceding application of Boltzmann's superposition principle was utilized in similar fashion (but with a constant applied, boundary deviatoric stress) to determine the time-dependent shear strain associated with dissolution of load bearing phases.

\section{Applications}

In order to illustrate the robustness and breadth of applicability of the kinematical framework and computationally implemented modeling approach, the apparent VE/VP creep behavior of two composite materials was simulated: 1) a porous solid containing ice in the pores that is gradually warmed and 2) hydrating cement paste. In the first case, creep occurs as a result of the melting process of the solid ice phases into the liquid water phases, which is functionally equivalent to the dissolution process in terms of the effect on stress redistributions. In this simulation case, the distribution of pore sizes plays a big role. Due to thermodynamic considerations (i.e., Gibbs-Thomson equation $[35,36]$ ), pores with different sizes melt at different temperatures. The melting temperature for larger pores is higher than for smaller pores, meaning that at a given relatively low temperature, smaller pores melt 
first while larger pores stay frozen. Thus, for a porous material starting at a very low temperature, all pores are filled with solid ice phases. When gradually warming the material while applying external mechanical loads to the porous structure, creep is induced as portions of load-bearing ice phases gradually melt. As the ice in the pores melts, whatever stress was carried by the ice is transferred to the surrounding, solid material.

The second simulation involves quantifying the creep that occurs in young cement paste that is undergoing the hydration reaction. When cement grains are mixed with water, a chemical reaction proceeds whereby the grains gradually dissolve and hydration products are precipitated out of the ion-rich aqueous solution. These products fill the space between the remaining cement grains and ultimately bond them together to form a solid-like matrix. If that matrix is stressed, both hydration products and remaining cement grains transmit a portion of the stress; as cement grains dissolve, the stress carried by those phases is transmitted to the remaining solid microstructure, which induces creep behavior in the composite.

\subsection{Creep due to the melting of ice within a porous material}

\subsubsection{Microstructure model}

Based on an algorithm previously utilized to generate random distributed impenetrable objects [37] and random dispersion of cement grains [38, 39], a Mathematica script was written to generate the 3D, cube shaped porous microstructures, which are shown in Fig. 4. These porous microstructures are solid matrices filled with randomly distributed pores, which are in the shape of spheres and are assigned with various radii. In this brute force algorithm, first, the largest pores are placed in the matrix in a cubic box with the dimension of $1 \mu \mathrm{m}$. Then, the second largest pores are randomly located in the matrix while avoiding any overlap with the previous existing pores. This procedure is repeated until a particular pore volume fraction is reached. The 3D images of the microstructures generated from the Mathematic file are then converted to grayscale 2D images. The domains are then subdivided into 100 pixels along each axis perpendicular to the faces of the cube (to form a total of $100 \times 100 \times 100=10^{6}$ voxels per microstructure per temperature step). Depending on the grayscale level of each voxel, every voxel inside the microstructure contains a single phase (solid/ice/water), which would be assigned mechanical properties in the finite element calculations. 


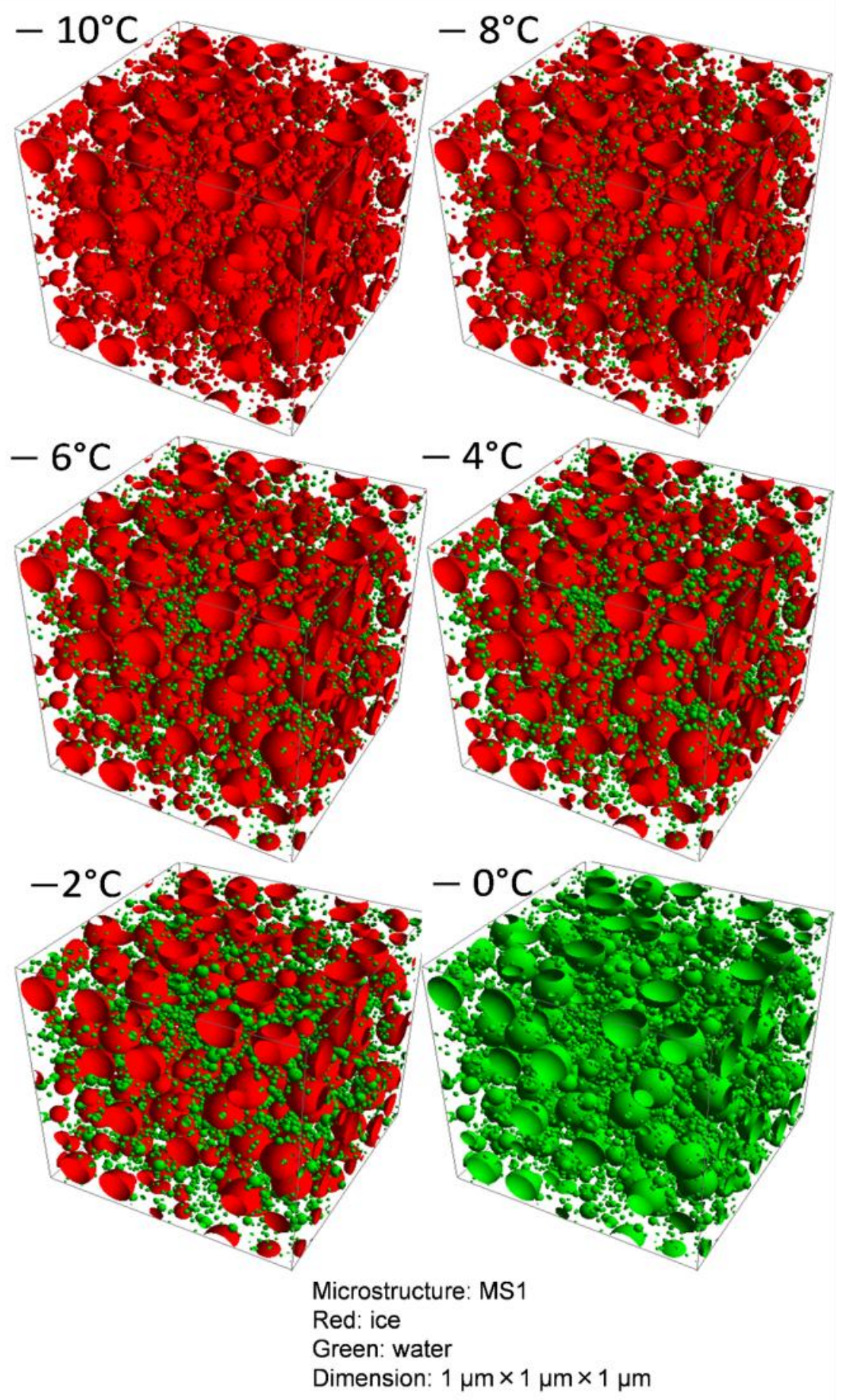

Fig. 4 Microstructure evolution of a frozen porous body with increasing temperature. The GibbsThomson equation was used to determine the limiting radius of the ice-filled pores at a given temperature. 
Two different series of microstructures were generated utilizing the aforementioned algorithm. Microstructure 1 (MS1) has a relatively narrow pore size distribution with the maximum pore size of $0.006 \mu \mathrm{m}$ and minimum pore size of $0.0062 \mu \mathrm{m}$. Microstructure 2 (MS2) has a wider and more uniform pore size distribution with the maximum pore size of 0.0034 $\mu \mathrm{m}$ and minimum pore size of $0.0227 \mu \mathrm{m}$. It can be easily seen that the pore sizes in MS1 are much larger than MS2. The porosity in MS1 is 0.2 , and the porosity in MS2 is 0.1 .

To determine the fraction of pores containing ice or water at each temperature for each microstructure, thermodynamic equilibrium was presumed to exist temporally at each time step. That is, the heating rate was slow enough so that each individual ice crystal could establish local equilibrium with its melt. As a consequence of the equilibrium between a solid crystal (e.g., ice) and its melt (e.g., liquid water), when temperature is increased, to stop ice from melting, the pressure in the crystal has to go down according to the relation

$$
p_{C}-p_{L}=\Sigma_{m}\left(T_{m}-T\right),
$$

where $p_{C}$ is the crystal pressure, $T$ is the current temperature, and the liquid pressure, $p_{L}$, remains at atmospheric pressure. $T_{m}$ and $\Sigma_{m}$ are the melting temperature and the melting entropy, respectively. The pressure difference, $p_{C}-p_{L}$ is related to the curvature radius, $R$, of the solid-liquid interface through the mechanical equilibrium (Laplace's equation),

$$
R=\frac{2 \gamma_{C L} \cos \theta}{p_{C}-p_{L}} .
$$

Here, $\theta$ is the contact angle between the liquid and the pore wall, and $\gamma_{C L}$ defines the crystal-liquid interface energy. Combining eqs. (22) and (23), the radius ${ }^{3}$ of the melting pore at a particular temperature can be determined as

$$
R=\frac{2 \gamma_{C L}}{\Sigma_{m}\left(T_{m}-T\right)} .
$$

That is, when the temperature increases to the current temperature, $T$, as given by (23), ice in all pores equal to and larger than the radius $R$ will melt. Eq. (23) is widely known as the Gibbs-Thomson equation.

\subsubsection{Simulation results}

Simulations are carried out on porous materials with time-evolving microstructures as ice melts inside its pore network utilizing the computational scheme. Two different factors are analyzed in the scheme to examine their effects on the overall creep behavior of ice-filled composites. One factor is different heating rates, which affects the melting/dissolution rate of the load bearing ice crystals, and the other one is different pore size distribution inherent to the microstructure. We choose to use the term 'dissolution' loosely here for the transition

\footnotetext{
${ }^{3}$ Here, for simplicity, we assume zero contact angle between the pore wall and the liquid water such that $R$, the radius of the crystal-liquid interface, coincides with the radius of the pore. This assumption is reasonable given that the contact angle of water with many materials is near zero.
} 
of ice to liquid water, since, from a stress-redistribution modeling perspective, removal of a solid due to phase change or actual dissolution in a solvent are functionally equivalent. In the simulations of the composites with frozen pores, the Young's modulus of ice phases was prescribed as $8.7 \mathrm{GPa}$, and the Poisson's ratio was set to be 0.31 [40], while the intrinsic creep of the ice was ignored. Meanwhile, a solid skeleton consisting of an isotropic material with elastic Young's modulus prescribed to be $24.9 \mathrm{GPa}$ and Poisson's ratio set to 0.15 was chosen. Poroelastic effects common in partially frozen materials (see, e.g., [41, 42]) were ignored; this means that liquid water inside the microstructure was assumed to be able to move freely and rapidly (versus the heating rate) to pressure free boundaries (e.g., nearby pores containing gases) and thus carry zero stress. From Fig. 5, an increasing axial strain of the composite as ice melts is quantified utilizing the new computational routine described in this paper. When the temperature increases from $-10^{\circ} \mathrm{C}$ to $0^{\circ} \mathrm{C}$, the evolution of axial strain of the composite structure is strongly affected by the different melting rates. The solid lines show the evolution of the stress-normalized axial creep strain of the composite under a slower rate of temperature increase $\left(1{ }^{\circ} \mathrm{C} / \mathrm{hr}\right)$ and thus a slower ice melting/dissolution rate, while dashed lines indicate a higher rate of temperature increase $\left(2^{\circ} \mathrm{C} / \mathrm{hr}\right)$ and thus a higher melting/dissolution rate. From Fig. 5, a higher melting rate (dissolution rate) would lead to faster creep of the composite. After the temperature reaches $0^{\circ} \mathrm{C}$, all the ice inside the microstructure has transitioned into water, and further increase in the temperature would not induce any additional creep behavior in the porous body.

(a)

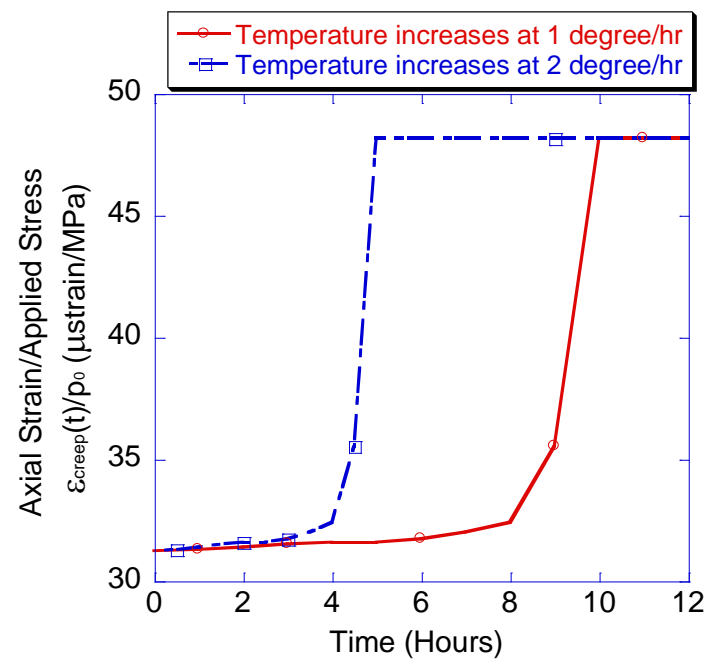

(b)

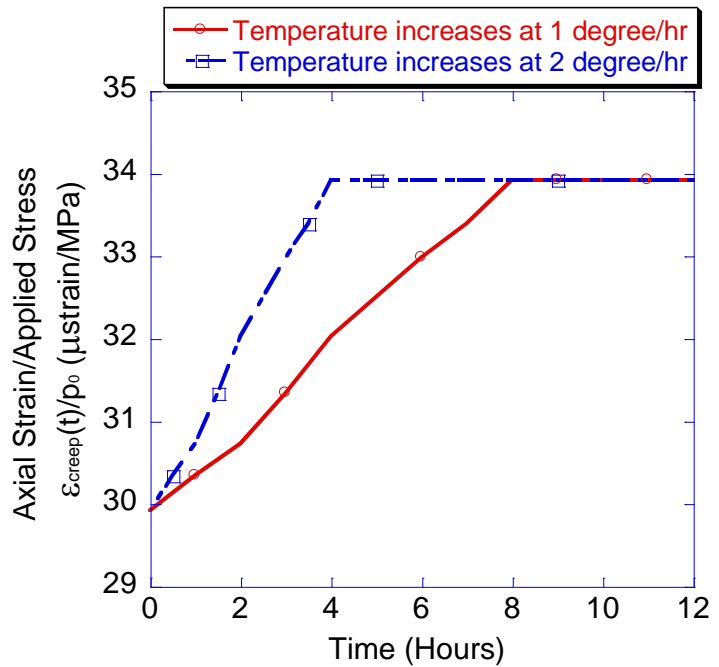

Fig. 5. Stress-normalized axial strain of a porous body with (a) large pore size distribution and (b) small pore size distribution under unit applied stress when the temperature of the microstructure increases from $-10^{\circ} \mathrm{C}$ to $0^{\circ} \mathrm{C}$ at different rates. In this graph, creep occurs strictly due to dissolution of load bearing ice phases. 


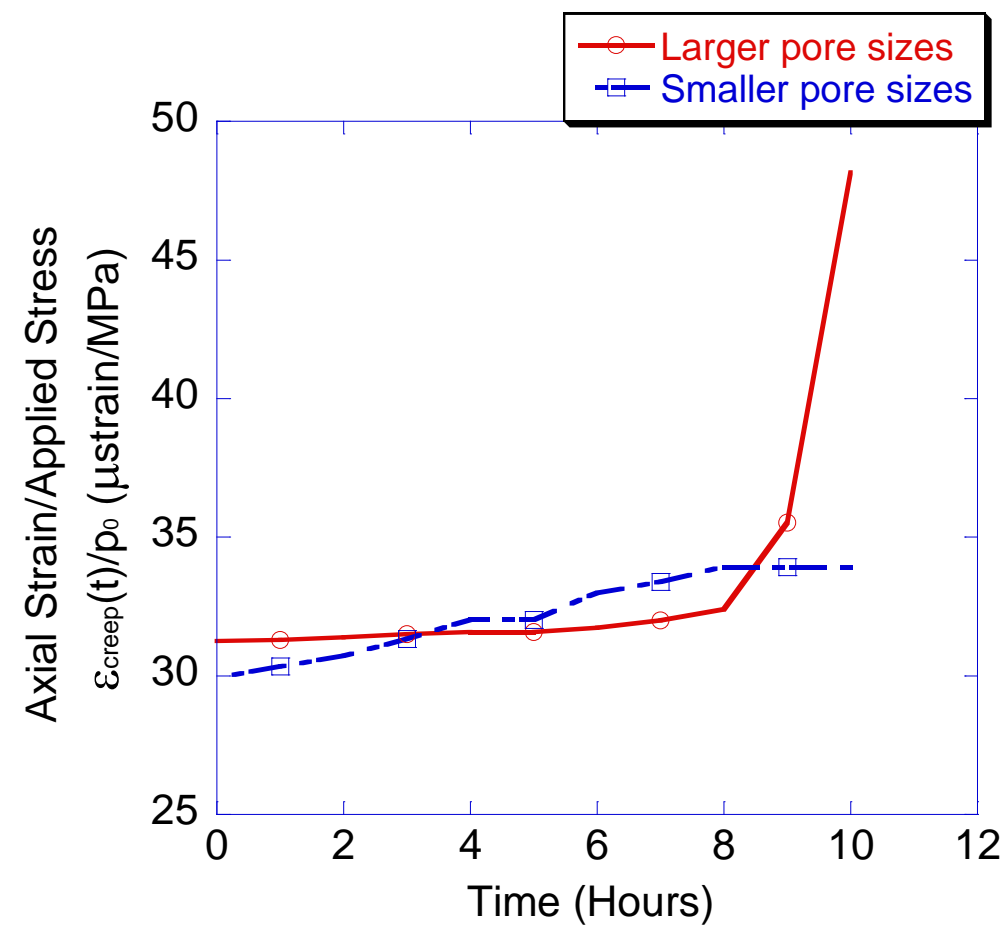

Fig. 6 Evolution of stress-normalized creep strain of a porous body with different pore size distributions under unit applied stress when the temperature of the microstructure increases from $10^{\circ} \mathrm{C}$ to $0^{\circ} \mathrm{C}$ at the rate of $1^{\circ} \mathrm{C} / \mathrm{h}$. In this graph, creep occurs strictly due to dissolution of load bearing ice phases.

The axial creep strain of porous bodies with different pore sizes is shown in Fig. 6 when the composites are subjected to warming (and thus dissolution of ice phases). From Fig. 6, when warming starts, the two composites establish similar instantaneous elastic deformation (because of similar elastic compliance) at time zero. As temperature increases, composites with different pore sizes establish different creep rates. The porous body with small, well distributed pore sizes creeps at a relatively constant rate over the time scale from $-10^{\circ} \mathrm{C}$ to $-2^{\circ} \mathrm{C}$, but the strain stays constant after the temperature reaches $-2^{\circ} \mathrm{C}$. The porous body with rather uniform, large pore sizes shows variable creep rate over the ice melting process. When the temperature rises from $-10^{\circ} \mathrm{C}$ to $-2^{\circ} \mathrm{C}$, the composite with large pore sizes creeps at a very slow rate. Once the temperature reaches $-2^{\circ} \mathrm{C}$ at the 8 th hour, suddenly, the large pore sizes composite shows a dramatic increase in the creep strain. The differences in creep rates are due to thermodynamic considerations; when an ice-filled porous body is subjected to increases in temperature, the melting rate of the ice phases is not purely linearly related to the rate of temperature change, but affected by the pore size distribution as well. Those materials with relatively well distributed pore sizes will have a roughly linear dependence of creep rate on rate of temperature increase. In contrast, those materials with a narrow, rather uniform pore size distribution will have a sudden large increase in creep strain when the temperature passes the threshold that induces melting of several of the pores simultaneously, 
which is what happens when the temperature reaches $-2^{\circ} \mathrm{C}$ (at about 9 hrs in Fig. 6) in the material with larger pore sizes.

One thing to notice here is that, in reality, as ice melts inside a porous body, the combined ice/liquid mixture would require less volume in the structure due to its increasing density. This may produce an inherent negative pressure inside the porous body, leading to potential contraction of the whole body as ice melts. Since this paper focuses on the investigations of the effect of solid phase dissolution (and thus stress redistribution) on the overall creep behavior of a frozen porous composite, a simplification is made in this paper that the creep behavior associated with the inherent pore pressurization (i.e., poromechanical effects [42$48]$ ) is neglected. Poromechanical effects may be significant in freezing materials if the pore fluid flow rate (controlled by material permeability, geometry, and pore fluid viscosity) is low relative to the freezing rate, and the pore fluid has low compressibility (of same order of magnitude or greater) than the porous body. For example, if pore fluid in a porous body contains any distributed entrapped air bubbles, then the pore fluid is highly compressible and poromechanical effects will be negligible during freezing events.

\subsection{Creep of cement paste due to chemical reaction induced dissolution}

\subsubsection{Microstructure model}

To generate the time-evolving microstructures for hydrating cement paste, a hydrationbased microstructural evolution model THAMES (Thermodynamic Hydration And Microstructure Evolution) $[49,50]$ was used. Based on empirically derived equations of clinker reaction rates [51] and on phenomenological dissolution kinetics [52], THAMES determines the effect of dissolving cement grains on the speciation of the pore fluid of reacting cement paste at evolving ages. Then, utilizing a thermodynamic engine GEMS (Gibbs Energy Minimization) [53, 54], which has been implemented into the THAMES, the thermodynamic equilibrium mass of the solid constituents, as well as the new equilibrium solution speciation can be calculated at each time step. In this way, THAMES is capable of simulating the hydration process of cement grains, and provides $3 \mathrm{D}$ images of the evolving cement paste at the micrometer level at different ages. A more detailed description of the microstructure model THAMES can be found in $[12,13,52]$. One thing to note about cement paste is that, when cement composites are subjected to external or internal stresses/strains, large local strain energy can be generated inside cement pastes. According to the second law of thermodynamics, this strain energy can potentially change the microstructure evolution of cement composites. Although the microstructure model THAMES can predict the microstructure evolution of cement paste during hydration process, it is not capable of accounting for the microstructure changes that occur due to applied stress/strain. Therefore, the simulation results generated in this paper on cement paste only considers the relaxation/creep behavior of cement composites as a result of cement grain dissolution during the hydration process, while the stress-induced microstructure change inside cement paste was disregarded in this paper. Furthermore, it is well established that C-S-H exhibits intrinsic viscoelasticity, which also can contribute to cement paste creep and relaxation. As the focus of this paper is to strictly demonstrate that evolving microstructures under load induce composite creep and relaxation, we consider here only creep and relaxation associated with the evolution of the cement microstructure associated with the hydration reaction. 


\subsubsection{Simulation results}

The creep behavior of $0.40 \mathrm{w} / \mathrm{c}$ cement paste at $298 \mathrm{~K}$ was simulated when subjected to a stress controlled boundary condition. All the phases in the cement paste were assumed to be purely elastic with assigned elastic properties taken from [52], so that all the VE/VP behavior of cement paste predicted in this section occurs strictly due to the dissolution effect of load-bearing cement grains. Two different loading ages $(1 \mathrm{~d}$ and $4 \mathrm{~d})$ were examined here to test the effect of different dissolution rate on the creep rate of cement paste. Fig. 7 shows the predicted creep of cement paste in axial strain while excluding the instantaneous elastic deformation of the composite under loading. In Fig. 7, cement paste continues to creep under constant applied stress as a result of the dissolution effect of loadbearing solid cement grains, and this effect is substantial. An older specimen would establish a slower creep rate because the hydration rate of cement grains at later ages is lower (and thus the dissolution rate). This prediction results successfully demonstrates the wellknown aging effect of cement paste whereby older specimens creep/relax slower than younger specimens (see, e.g. [55, 56]). When C-S-H (calcium silicate hydrate) viscoelasticity has been traditionally considered as the major mechanism leading to cement paste creep/relaxation, the solidification theory developed by Bazant has often been utilized in predicting the time-dependent behavior of cement paste or concrete [57]. However, in the solidification theory, it is presumed that cement grains cannot transmit stress. As a result of this presumption, under a constant macroscopic stress, the average stress in the solidifying material (including C-S-H) is predicted to decay with age as the volume of such material progressively increases. This decay in average stress in the solidifying material yields the aging effect observed in experiments on creep of cementitious materials. In contrast, in the modeling approach herein the cement grains are allowed to transmit stress, which results in an increase in the average stress in the solidifying phases as cement grains dissolve. The aging effect arises primarily due to gradual reductions in the rate of cement grain dissolution with age. More detailed comparisons between the solidification theory with the computational scheme can be found in [58]. 


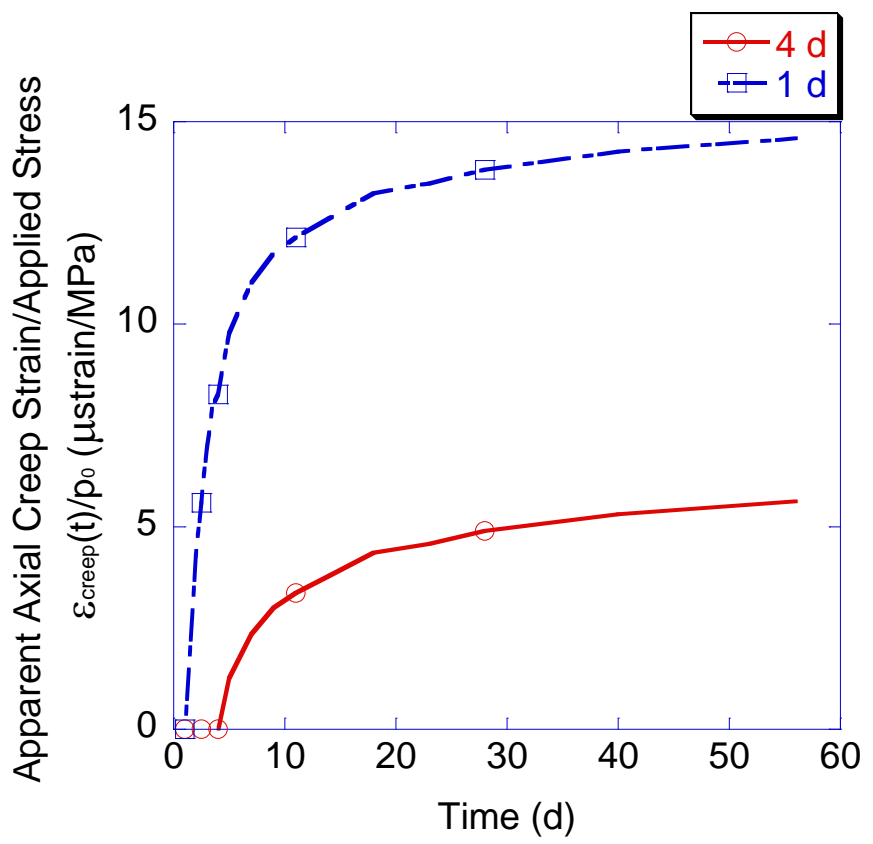

Fig. 7. Apparent axial creep strain of $0.40 \mathrm{w} / \mathrm{c}$ cement paste when subject to unit stress at different ages $(1 d$ and $4 d)$. In this graph, creep occurs strictly due to dissolution of load bearing cement grains.

\section{Conclusions}

A kinematic framework was established to quantify the effect of dissolution of load-bearing phases on the overall stress and strain fields in evolving composite materials. The framework accounts for the differing evolutions of natural configurations in a particular phase (existing in a particular voxel) in the discretized microstructure and in the composite material body. Based on the developed framework, a microstructure evolution model and a time-stepping finite element method capable of tracking phase formation were integrated to create a computational scheme. The kinematic framework and complementary computational approach provide a methodology by which stress relaxation or creep of any composite body with dissolving phases may be modeled. To demonstrate this utility, the approach was applied to two different composite materials to predict their apparent timedependent creep behavior when subjected to stress controlled boundary conditions. When simulating the apparent creep strain of a water saturated, frozen, porous body due to dissolution of ice phases upon warming, the model captures the effect of heat rate and the effect of pore size distribution. And when predicting the apparent VE/VP behavior of early-age cement paste, the well-known aging effect is successfully demonstrated. From both the simulation results, the effect of dissolution of solid, load-bearing phases in leading to apparent time-dependent behavior is substantial.

While the kinematic framework is established with no restrictions regarding the magnitude of the deformation gradient, the computational scheme utilizing the framework presented here is limited to small deformation gradients. Thus, the computational scheme is currently 
limited to predicting composite behavior at stress magnitudes below that which induce locally large strains. For the cementitious materials simulated in this paper, such a limit restricts the simulations to stress levels that do not induce local damage.

\section{Acknowledgments}

This research was financially supported by the US National Science Foundation via grant numbers 0843979 and 1327314. Any opinions, findings, and conclusions or recommendations expressed in this material are those of the author(s) and do not necessarily reflect the views of the National Science Foundation. The authors also thank Edward Garboczi, Jeffrey Bullard, and Kumbakonam Rajagopal for their invaluable discussions and suggestions.

\section{References}

1. Aydin, A.C., A. Arslan, and R. Gül, Mesoscale simulation of cement based materials' time-dependent behavior. Computational Materials Science, 2007. 41(1): p. 20-26.

2. Jeon, J., J. Kim, and A. Muliana, Modeling time-dependent and inelastic response of fiber reinforced polymer composites. Computational Materials Science, 2013. 70: p. 37-50.

3. Drozdov, A.D., E.A. Jensen, and J.d.C. Christiansen, Nonlinear time-dependent response of polypropylene/nanoclay melts: Experiments and modeling. Computational Materials Science, 2010. 47(3): p. 807-816.

4. Suter, M. and G. Benipal, Constitutive model for aging thermoviscoelasticity of reacting concrete I: theoretical formulation. Mechanics of Time-Dependent Materials, 2010. 14(3): p. 277-290.

5. Rutter, E. and D. Elliott, The kinetics of rock deformation by pressure solution [and discussion]. Philosophical Transactions of the Royal Society of London A: Mathematical, Physical and Engineering Sciences, 1976. 283(1312): p. 203-219.

6. Rutter, E., Pressure solution in nature, theory and experiment. Journal of the Geological Society, 1983. 140(5): p. 725-740.

7. Weyl, P.K., Pressure solution and the force of crystallization: a phenomenological theory. Journal of Geophysical Research, 1959. 64(11): p. 2001-2025.

8. Ghoussoub, J. and Y.M. Leroy, Solid-fluid phase transformation within grain boundaries during compaction by pressure solution. Journal of the Mechanics and Physics of Solids, 2001. 49(10): p. 2385-2430.

9. Niemeijer, A.R., C.J. Spiers, and B. Bos, Compaction creep of quartz sand at 400600C: experimental evidence for dissolution-controlled pressure solution. Earth and Planetary Science Letters, 2002. 195(3-4): p. 261-75.

10. Shimizu, I., Kinetics of pressure solution creep in quartz; theoretical considerations. Tectonophysics, 1995. 245(3-4): p. 121-134.

11. Karato, S.-I. and H. Jung, Effects of pressure on high-temperature dislocation creep in olivine. Philosophical Magazine, 2003. 83(3): p. 401-414.

12. Li, X., et al., Computing the time evolution of the apparent viscoelastic/viscoplastic Poisson's ratio of hydrating cement paste. Cement and Concrete Composites, 2015. 56: p. 121-133. 
13. $\mathrm{Li}, \mathrm{X}$., et al., Computing the time evolution of the apparent viscoelastic/viscoplastic poisson's ratio of hydrating cement paste. Cement and Concrete Composites, 2015. 56: p. 121-133.

14. Li, X., S. Rahman, and Z.C. Grasley, Modeling Creep and Relaxation Caused by Phase Dissolution, in Challenges in Mechanics of Time Dependent Materials, Volume 2, B. Antoun, Editor. 2016, Springer International Publishing. p. 73-81.

15. Villani, A., E.P. Busso, and S. Forest, Field theory and diffusion creep predictions in polycrystalline aggregates. Modelling and Simulation in Materials Science and Engineering, 2015. 23(5): p. 055006.

16. Ruetz, W. A hypothesis for the creep of hardened cement paste and the influence of simultaneous shrinkage. in Proceedings of International Conference On the Structure of Concrete. 1968. London, England: Cement and Concrete Association.

17. Kannan, K. and K.R. Rajagopal, A thermodynamical framework for chemically reacting systems. Zeitschrift fur Angewandte Mathematik und Physik, 2011. 62(2): p. 331-363.

18. Rajagopal, K.R. and A.R. Srinivasa, On the thermomechanics of materials that have multiple natural configurations Part I: Viscoelasticity and classical plasticity. Zeitschrift für angewandte Mathematik und Physik ZAMP, 2004. 55(5): p. 861893.

19. Rao, I.J. and K.R. Rajagopal, A thermodynamic framework for the study of crystallization in polymers. Zeitschrift für angewandte Mathematik und Physik ZAMP, 2002. 53(3): p. 365-406.

20. Rajagopal, K.R. and A.S. Wineman, A note on viscoelastic materials that can age. International Journal of Non-Linear Mechanics, 2004. 39(10): p. 1547-1554.

21. Lapczyk, I., K.R. Rajagopal, and A.R. Srinivasa, Deformation twinning during impact - numerical calculations using a constitutive theory based on multiple natural configurations. Computational Mechanics, 1998. 21(1): p. 20-27.

22. Lapczyk, I., K.R. Rajagopal, and A.R. Srinivasa, Deformation twinning during impact of a titanium cylinder - numerical calculations using a constitutive theory based on multiple natural configurations. Computer Methods in Applied Mechanics and Engineering, 2000. 188(1): p. 527-541.

23. Rajagopal, K.R. and A.R. Srinivasa, Modeling anisotropic fluids within the framework of bodies with multiple natural configurations. Journal of NonNewtonian Fluid Mechanics, 2001. 99(2-3): p. 109-24.

24. Rajagopal, K.R. and A.R. Srinivasa, On the thermomechanics of materials that have multiple natural configurations: viscoelasticity and classical plasticity. Zeitschrift fur Angewandte Mathematik und Physik, 2004. 55(5): p. 861-93.

25. Rajagopal, K.R. and A.R. Srinivasa, On the thermomechanics of materials that have multiple natural configurations: twinning and solid to solid phase transformation. Zeitschrift fur Angewandte Mathematik und Physik, 2004. 55(6): p. 1074-93.

26. Rajagopal, K.R. and A.R. Srinivasa, On the role of the Eshelby energy-momentum tensor in materials with multiple natural configurations. Mathematics and Mechanics of Solids, 2005. 10(1): p. 3-24. 
27. Ravindran, P., et al., Modelling sand-asphalt mixtures within a thermodynamic framework: Theory and application to torsion experiments. International Journal of Pavement Engineering, 2009. 10(2): p. 115-131.

28. Mura, T., General theory of eigenstrains, in Micromechanics of defects in solids. 1987, Springer Netherlands: Dordrecht. p. 1-73.

29. Haecker, C.J., et al., Modeling the linear elastic properties of Portland cement paste. Cement and Concrete Research, 2005. 35(10): p. 1948-60.

30. Garboczi, E.J., Finite element and finite difference programs for computing the linear electric and elastic properties of digital images of random materials. 1998, Building and Fire Research Laboratory,National Institute of Standards and Technology.

31. Bohn, R.B. and E.J. Garboczi, User manual for finite element and finite difference programs: A parallel version of NISTIR-6269. 2003, Gaithersburg: U.S. Department of commerce, Technology Administration, National Institute of Standards and Technology, Information Technology Laboratory, Building and Fire Research Labortory.

32. Zohdi, T.I., Homogenization Methods and Multiscale Modeling, in Encyclopedia of Computational Mechanics. 2004, John Wiley \& Sons, Ltd.

33. Wineman, A.S. and K.R. Rajagopal, Mechanical response of polymers: an introduction. 2000: Cambridge University Press.

34. Christensen, R., Theory of viscoelasticity: an introduction. 2012: Elsevier.

35. Petrov, O. and I. Furó, Curvature-dependent metastability of the solid phase and the freezing-melting hysteresis in pores. Physical Review E, 2006. 73(1): p. 011608 .

36. Strange, J.H., M. Rahman, and E. Smith, Characterization of porous solids by NMR. Physical review letters, 1993. 71(21): p. 3589.

37. Bentz, D., E. Garboczi, and K. Snyder, Hard Core/Soft Shell Microstructural Model for Studying Percolation and Transport in Three-Dimensional Composite Media. 1999. p. 55.

38. Yazdanbakhsh, A., Production, Characterization, and Mechanical Behavior of Cementitious Materials Incorporating Carbon Nanofibers. 2012, Texas A\&M University.

39. Yazdanbakhsh, A. and Z. Grasley, The theoretical maximum achievable dispersion of nanoinclusions in cement paste. Cement and Concrete Research, 2012. 42(6): p. 798-804.

40. Hobbs, P.V., Ice Physics, ed. O.C.T.i.t.P. Sciences. 2010: Oxford University Press.

41. Rahman, S., et al., Simulation of Mass, Linear Momentum, and Energy Transport in Concrete with Varying Moisture Content during Cooling to Cryogenic Temperatures. Transport in Porous Media, 2016. DOI: 10.1007/s11242-016-06368.

42. Rahman, S. and Z. Grasley, A poromechanical model of freezing concrete to elucidate damage mechanisms associated with substandard aggregates. Cement and Concrete Research, 2014. 55(0): p. 88-101.

43. Leung, C.K. and Z.C. Grasley, Poromechanical Damping of Cementitious Materials. Journal of Materials in Civil Engineering, 2012. 24(2): p. 232-238. 
44. Jones, C.A. and Z.C. Grasley, Correlation of Radial Flow-Through and Hollow Cylinder Dynamic Pressurization Test for Measuring Permeability. Journal of Materials in Civil Engineering, 2009. 21(10): p. 594-600.

45. Jones, C.A. and Z.C. Grasley, Correlation of hollow and solid cylinder dynamic pressurization tests for measuring permeability. Cement and Concrete Research, 2009. 39(4): p. 345-352.

46. Jones, C.A. and Z.C. Grasley, Novel and flexible dual permeability measurement device for cementitious materials. ACI Materials Journal, 2009. 106(2): p. 192-197.

47. Jones, C.A. and Z.C. Grasley. Measuring concrete permeability using dynamic pressurization: achieving saturation. in 2009 NRMCA Concrete Technology Forum. 2009. Cincinnati, OH: National Ready Mix Concrete Association.

48. Rahman, S. and Z. Grasley, Determining optimum air-void spacing requirement for a given concrete mixture design using poromechanics. International Journal of Pavement Engineering, 2016: p. 1-10.

49. Landau, L.D. and E.M. Lifshitz, Theory of elasticity. 3rd ed. 1986, Oxford: Pergamon.

50. Bentz, D., E. Garboczi, and K. Snyder, Hard Core/Soft Shell Microstructural Model for Studying Percolation and Transport in Three-Dimensional Composite Media. 1999. p. 55.

51. Parrot, L. and D. Killoh. Prediction of cement hydration. in Proc. Br. Ceram. Soc. 1984.

52. Bullard, J.W., et al., Coupling thermodynamics and digital image models to simulate hydration and microstructure development of portland cement pastes. Journal of Materials Research, 2011. 26(4): p. 609-622.

53. Lothenbach, B. and F. Winnefeld, Thermodynamic modelling of the hydration of portland cement. Cement and Concrete Research, 2006. 36(2): p. 209-226.

54. Parrot, L. and D. Killoh. Prediction of cement hydration. in Proceedings of the British Ceramic Society. 1984.

55. Grasley, Z.C. and D.A. Lange, Constitutive modeling of the aging viscoelastic properties of portland cement paste. Mechanics of Time-Dependent Materials, 2007. 11(3-4): p. 175-198.

56. Rossi, P., J.-L. Tailhan, and F. Le Maou, Comparison of concrete creep in tension and in compression: Influence of concrete age at loading and drying conditions. Cement and Concrete Research, 2013. 51: p. 78-84.

57. Carol, I. and Z.P. Bazant, Viscoelasticity with aging caused by solidification of nonaging constituent. Journal of Engineering Mechanics, ASCE, 1993. 119(11): p. 2252-2269.

58. Li, X., et al., Modeling the apparent and intrinsic viscoelastic relaxation of hydrating cement paste. Cement and Concrete Composites, 2015. 55: p. 322-330. 

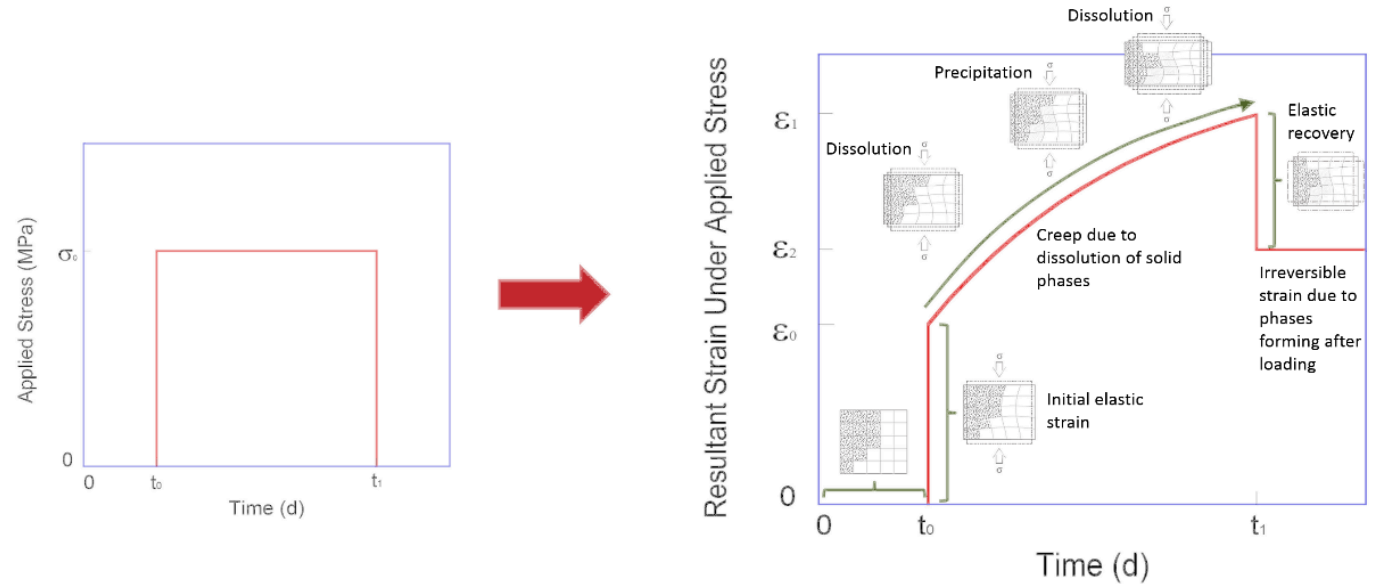